\title{
The tilted disc
}

\section{DAVID DORRELL}

From the Department of Neuro-Ophthalmology, National Hospitals for Nervous Diseases, Queen Square, London

SUMMARY Sixty tilted discs were studied by colour photography, including some by fluorescein angiography. Attention was drawn to the contracted, D-shaped outline of the scleral canal, and it was suggested that fewer fibres than normal enter the defective side of the disc. This was supported by examination of the nerve fibre layer and the discovery of field defects in 13 of the 27 eyes in which the visual fields were examined. The similarity of these features with congenital hypoplasia of the optic nerve head was noted.

The tilted disc is a congenital anomaly of the optic nerve head. Other terms used to describe discs with a similar appearance are Fuchs's coloboma, congenital crescents, conus, dysversion of the optic nerve head, and situs inversus. An incidence of $3.41 \%$ in 32522 eyes was reported by von Szily (1922) in a study of congenital crescents excluding those on the temporal side.

The object of this paper is to discuss the features of the tilted disc, to clarify its nomenclature, and to speculate briefly on its aetiology. Frequent accounts, for example, by Rucker (1946), Graham and Wakefield (1973), Manor (1974), and Young et al. (1976), have already drawn attention to the association of this anomaly with astigmatism and field defects, especially of a bitemporal character.

\section{Methods}

Sixty optic discs were examined by colour photography, aided in certain cases by fluorescein angiograms and stereoscopic photographs. In 27 eyes the refraction was measured and the visual fields plotted, care being taken to eliminate refractive scotomas where necessary.

\section{Results}

There were 3 salient features of this congenital abnormality-the crescent, the depression of one side of the disc, and the oblique direction of the retinal vessels as they entered the eye. One, two, or all of these features may be seen to a varying degree.

Address for reprints: David Dorrell, FRCS, Department of Neuro-Ophthalmology, National Hospitals for Nervous Diseases, Queen Square, London WCIN 3BG
This study was limited to those discs in which all 3 defects were unquestionably present.

\section{THE CRESCENT}

This was created by a disparity between the size of the retinal and scleral openings at the optic nerve head (Fig. 1). It appeared that in every case the retinal opening was approximately circular in shape and normal in size, whereas the outline of the scleral canal was contracted across 1 segment, so

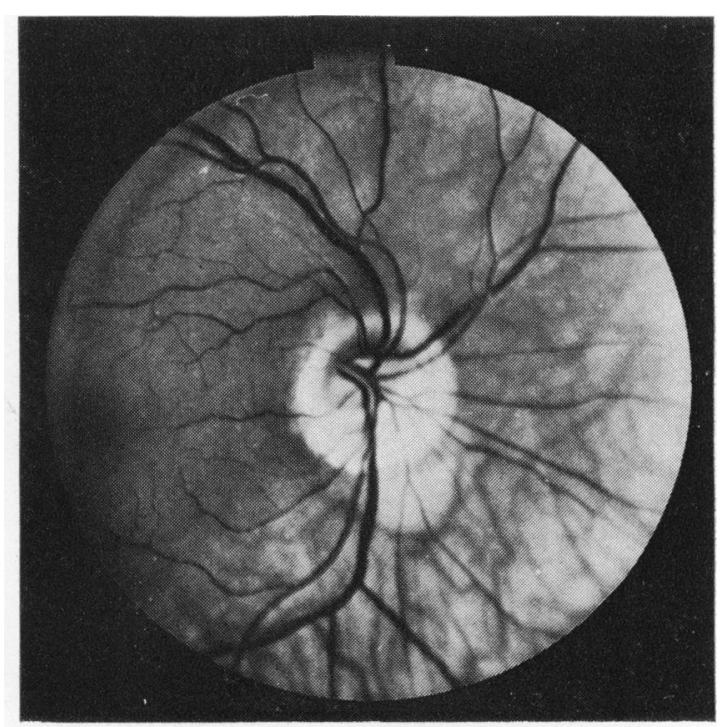

Fig. 1 The disc is apparently tilted downwards. The upper vessels curve over the retina and choroid, while below the vessels run over the straight edge of the $D$-shaped scleral opening and, before reaching the retina, pass across the exposed sclera 
that it conformed to a stirrup or D-shape (Fig. 2).

This distinguished the congenital crescent from the acquired and progressive type, found in the expanding myopic eye, where the edge of the retina is pulled away from the temporal side of the scleral canal.

In some eyes the whole disc assumed a D-shaped appearance. The crescent and the border of the

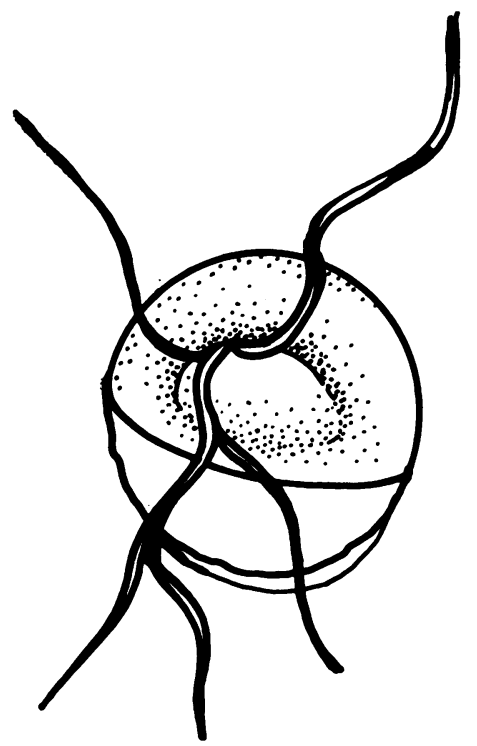

Fig. 2 The incongruity between the D-shaped scleral canal (stippled) and the circular retinal opening forms the crescent retina were difficult to discern, but they became obvious during fluorescein angiography when the rim of pigment epithelium was outlined (Fig $3 a, b$ ). In other cases the D-shaped cross-section of the scleral canal was barely visible beneath the nerve fibre layer. Once again it was readily betrayed by fluorescein angiography, which showed diminished capillary filling over the abnormal segment of the disc (Fig. 4a, b).

TILTING

The defective edge of the optic disc was depressed, since within the crescent both retina and choroid were absent, leaving only a thin covering of nerve fibres over the sclera. Conversely, the opposing border of the disc, composed of 3 layers of normal retina, gave an impression of elevation. Moreover, the crowding of axons over the upper edge of the disc in 4 patients led to an erroneous impression of papilloedema (Fig. 5).

Thus the discrepancy in the height between opposite sides of the disc gave rise to the tilted appearance. This was emphasised in many cases by a reduction in the quantity of nerve fibres passing over the lower edge.

The direction of the tilt was to the nasal side of the vertical meridian in 36 eyes $(60 \%)$, downwards in $7(11.7 \%)$, and infero-temporally in $17(28 \cdot 3 \%)$.

\section{NER VE FIBRE LAYER}

The visual assessment of the density of axons at the optic disc is liable to criticism, yet with the aid of intense red-free light and both colour and

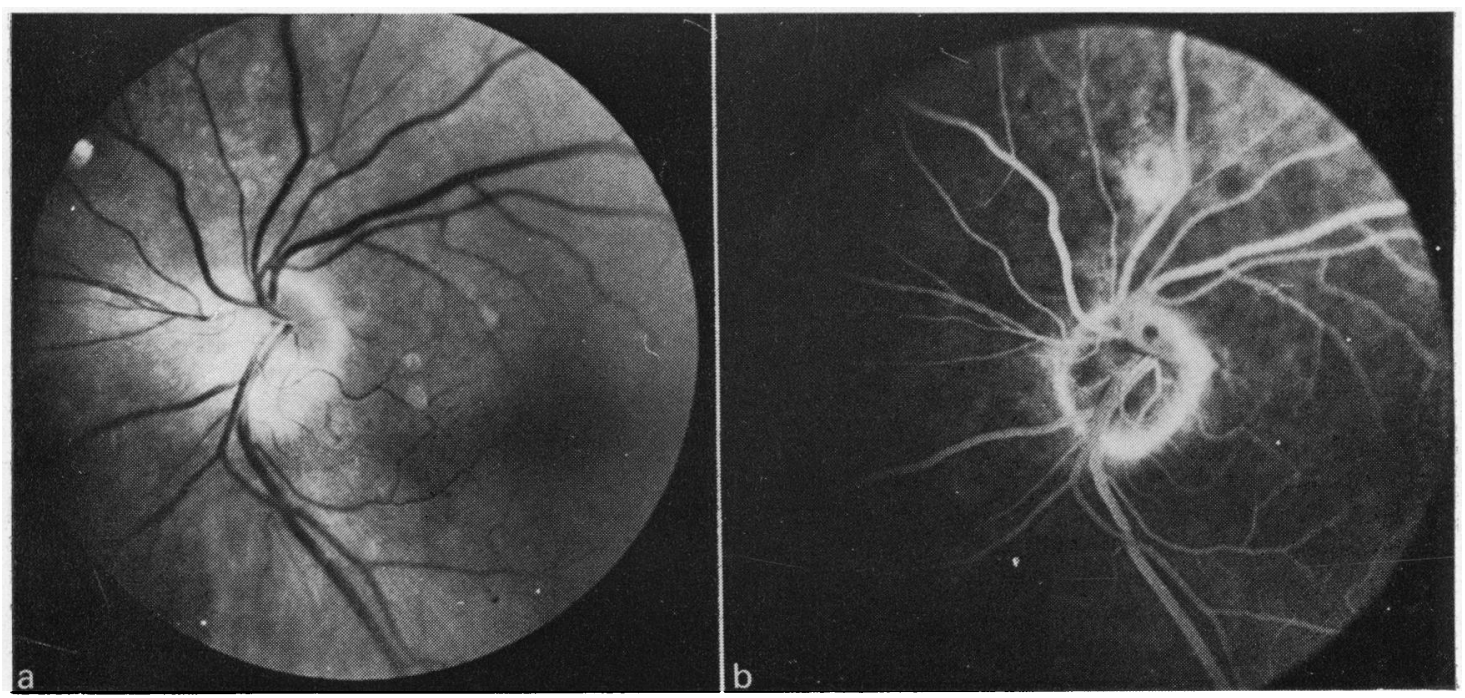

Fig. $3 a, b$ In some cases the circular retinal opening is invisible ophthalmoscopically (a), but after fluorescein injection the edge of the pigment epithelium is outlined (b) 

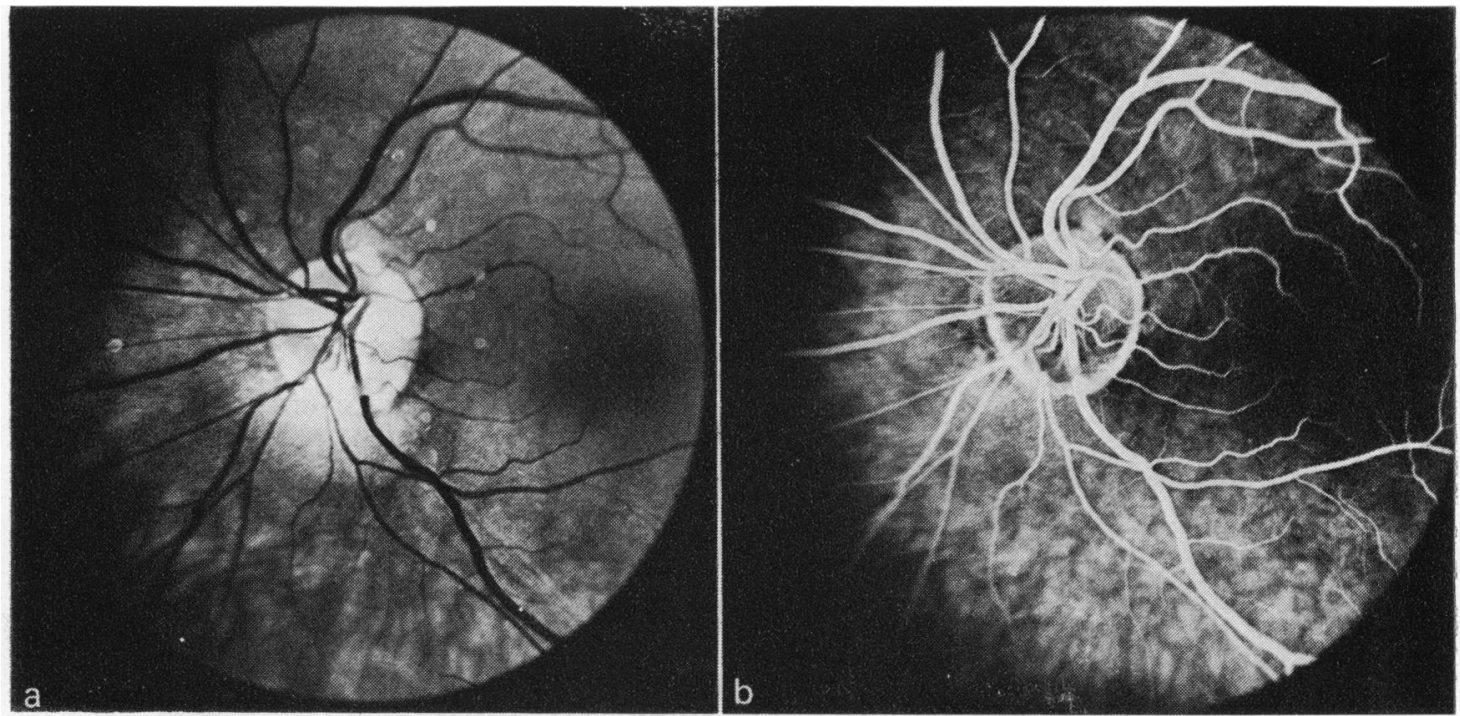

Fig. $4 a, b$ The postinjection photograph (b) shows reduced filling over the area of the crescent

stereoscopic photographs fewer fibres appeared to enter the optic nerve head in the sector related to the crescent in many of the eyes which were examined (Fig. 5).

Defects in the visual field provided further evidence of diminished axons. They corresponded to the direction of the tilt, and an upper-half scotoma

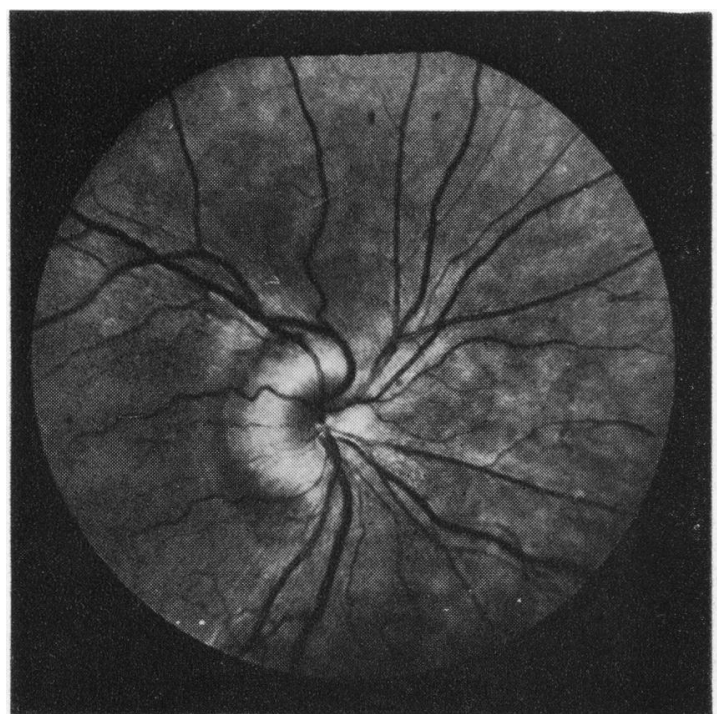

Fig. 5 The appearance of pseudopapilloedema is given by the nerve fibres from the superior and temporal retina, which crowd into a scleral canal of diminished size. They contrast with the paucity of fibres crossing the abnormal inferonasal segment of the disc was demonstrated in 13 of the 27 eyes in which the visual fields were plotted.

\section{VESSEL ENTRY}

In the normal disc the retinal vessels enter the eye in an axial direction. In those which were tilted the vessels were deviated towards the crescent, thus enhancing the tilted appearance.

FUNDUS PIGMENT

In $46(76.7 \%)$ of the 60 eyes there was less pigment in a wedge-shaped area of the fundus below the nerve head (Fig. 6). This was seen in 11 of 13 eyes which showed defects in the visual field. In the remaining 2 eyes a generalised reduction in fundus pigment was found.

\section{Discussion}

\section{FEATURES}

The most significant feature of the tilted disc is the D-shape of the scleral opening; it creates the crescent of uncovered sclera between the canal and the normal circular opening in the retina.

Recent reports scarcely allude to this deformity of the scleral canal, although it is beautifully illustrated by von Szily (1922), and Cacamise (1954) describes a horizontal edge between crescent and disc. Both Mann (1922) and Duke-Elder (1964) attribute the crescent to a deficiency of the choroid, neural retina, and pigment epithelium, which fails to indent the optic nerve. Furthermore, Young et al. (1976) in their histological study report that the pigment 


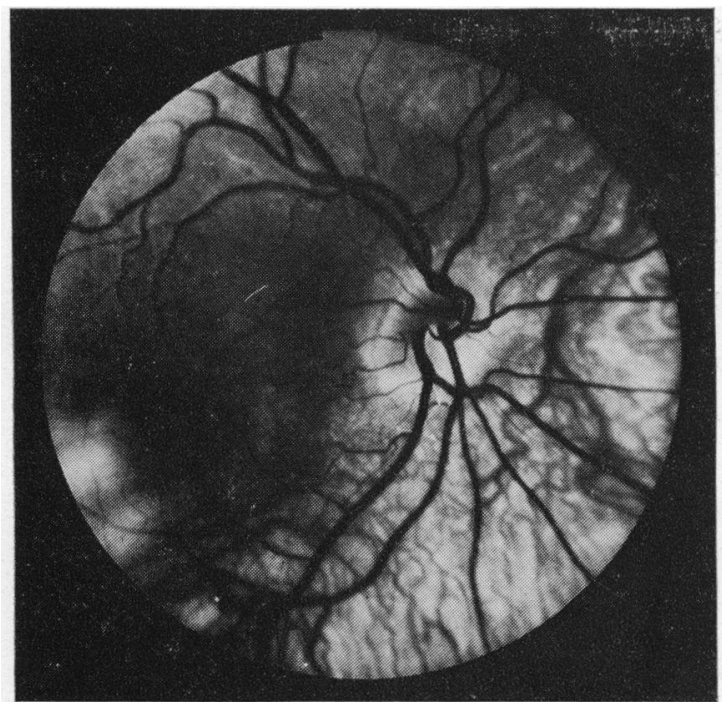

Fig. 6 There is a relative lack of pigment in the sector of the fundus associated with the defective segment of the disc

epithelium and choroid are displaced away from the disc. These opinions, which conflict with the concept of a defective scleral canal, could be explained by the difficulty in distinguishing a small scleral opening from a large retinal opening on a vertical cross-section of the optic disc (Fig. 7).

It is this contraction of the scleral canal which forms a resemblance to congenital hypoplasia of the optic nerve head, although only over a small segment of the disc's circumference. Moreover the double-ringed appearance of the hypoplastic disc, caused by the disparity between scleral and retinal openings, relates to the crescent of the tilted disc. The paucity of fibres entering the nerve is another feature shared by these congenital anomalies. Probably all tilted discs carry fewer axons than normal, although this diminution may be insufficient to lead to a defect in the visual field when carefully plotted by Goldmann perimetry.

Why should the retinal vessels follow an oblique direction of entry? The nerve fibres which pass over the normal edge of the disc not only are more numerous but need to effect a sharp turn to enter the scleral canal; in contrast the fewer fibres from the lower fundus need only follow a gentle curve over the abnormal edge of the disc, which is depressed and sloping. Thus the vessels, closely related to the nerve fibres, are swept down towards the crescent as they pass into the eye.

\section{NOMENCLATURE}

The depression of one side of the optic disc and the oblique direction of vessel entry give rise to the terms tilted, dysversion of the optic nerve head, and situs inversus. The third feature of the tilted disc, the crescent, accounts for the names conus, congenital crescent, and Fuchs's coloboma.

Until there is stronger evidence for the hypoplastic segment in these discs the purely descriptive term of 'tilted' would seem the most appropriate. It is preferred to the others because the crescent or conus is not always easy to discern, while one is often visible on the temporal side in the normal disc.

According to Duke-Elder (1964) dysversion of the optic nerve head results in tilting of the optic disc to a less dramatic degree than produces a crescent. This description could not be applied to our group of discs, since they all had crescents caused by the D-shaped scleral canal. Nor was the term situs inversus apt, because none of our tilted discs were pointed nasally along the horizontal meridian, although some approached to within $20^{\circ}$ of it. However, $60 \%$ were directed into the inferonasal quadrant.

The title Fuchs's coloboma has a deeper implication, which has yet to be substantiated.

\section{AETIOLOGY}

In analysing these patients our attention was drawn to the following points.

The deficit in nerve fibres, passing over the crescent, may result from a failure of axons to develop from a defective segment of the lower retina; alternatively, the fibres may grow normally down the optic stalk, only to atrophy at a later date.

The sclera is first laid down at the limbus, but does not reach the optic stalk and form the scleral

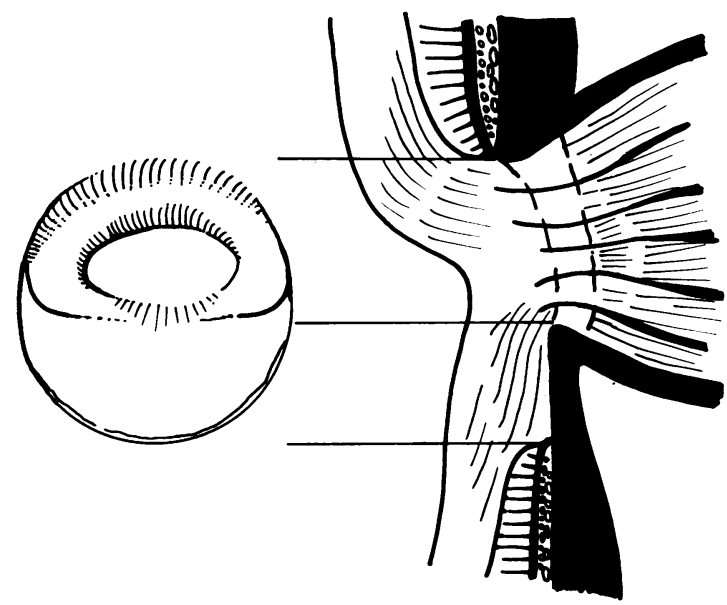

Fig. 7 In a vertical cross-section of a tilted disc it is difficult to distinguish a small scleral opening from an enlarged retinal opening 
canal until the fifth month; this succeeds the entry of nerve fibres into the optic stalk by several weeks. The normal circular outline of the canal might perhaps be curtailed if there were a defect in the optic nerve or a failure of the embryonic fissure to close where the optic stalk joins the optic cup.

The embryonic fissure closes at the sixth week in the inferior nasal quadrant. The prevalence of diminished pigmentation in the segment of the fundus related to the tilt, and its frequent association with field defects, suggest a relationship to an anomaly in closure of the fissure. All the cases with field defects reported by Young et al. (1976) showed hypopigmentation inferonasally. Also in all our cases the tilt was downwards in direction, though not always in the inferonasal quadrant of the embryonic fissure.

Mann (1922) related the inferior crescent to an eversion of the inner layer of the optic cup during closure of the embryonic fissure. This phenomenon occurs normally in the bird.

Peterson and Walton (1977) reported 17 children, born of severely diabetic mothers, who had what they described as segmental optic nerve hypoplasia with good visual acuity but with altitudinal or sector field defects. It was their supposition that there is a relationship between maternal diabetes and segmental hypoplasia of the optic nerve.

We have remarked on the similarity between segmental hypoplasia of the disc and tilting of the disc, so that maternal diabetes may be another possible aetiological factor in the latter.

I am grateful to Mr S. J. Miller and Mr M. D. Sanders for allowing me to report these cases, and to Mr Rolf Sennhenn for providing the photographs.

\section{References}

Cacamise, W. C. (1954). Situs inversus of the optic disc with inferior conus and variable myopia. A case report. American Journal of Ophthalmology, 38, 854-856.

Duke-Elder, S. (1964). Congenital Deformities. In System of Ophthalmology, vol. 3, Part 2, p. 674. Kimpton: London.

Graham, M. V., and Wakefield, G. S. (1973). Bitemporal field defects associated with anomalies of the optic discs. British Journal of Ophthalmology, 57, 307-314.

Mann, I. (1922). Developmental Anomalies of the Eye, 2nd edition, pp. 100-113. British Medical Association: London.

Manor, R. S. (1974). Temporal field defects due to nasal tilting of discs. Ophthalmologica, 168, 269-281.

Peterson, R. A., and Walton, D. S. (1977). Optic nerve hypoplasia with good visual acuity and visual field defects. Archives of Ophthalmology, 95, 254-258.

Rucker, C. W. (1946). Bitemporal defects in the visual fields resulting from developmental anomalies of the optic discs. Archives of Ophthalmology, 35, 546-554.

von Szily, A. (1922). Uber den Conus in heterotypisher Richtung. Albrecht von Graefes Archiv für Ophthalmologie, 110, 183-291.

Young, S. E., Walsh, F. B., and Knox, D. L. (1976). The tilted disc syndrome. American Journal of Ophthalmology, 82, 16-23. 